\title{
Global Existence and Extinction of Weak Solutions to a Class of Semiconductor Equations with Fast Diffusion Terms
}

\author{
Bin Wu \\ Department of Information and Computing Sciences, College of Mathematics and Physics, \\ Nanjing University of Information Science and Technology, Nanjing 210044, China \\ Correspondence should be addressed to Bin Wu, wubing790831@126.com
}

Received 2 March 2008; Accepted 13 August 2008

Recommended by Y. Giga

We consider the transient drift-diffusion model with fast diffusion terms. This problem is not only degenerate but also singular. We first present existence result for general nonlinear diffusivities for the Dirichlet-Neumann mixed boundary value problem. Then, the extinction phenomenon of weak solutions for the homogeneous Dirichlet boundary problem is studied. Sufficient conditions on the extinction and decay estimates of solutions are obtained by using $L^{p}$-integral model estimate method.

Copyright (C) 2008 Bin Wu. This is an open access article distributed under the Creative Commons Attribution License, which permits unrestricted use, distribution, and reproduction in any medium, provided the original work is properly cited.

\section{Introduction}

The scaled semiconductor drift-diffusion model reads

$$
\begin{gathered}
-\nabla \cdot(\nabla \psi)=p-n+C(x), \\
n_{t}-\nabla \cdot J_{n}=r(n, p)(1-n p)+g, \quad J_{n}=\left(\nabla\left(n^{m}\right)-n \nabla \psi\right), \\
p_{t}+\nabla \cdot J_{p}=r(n, p)(1-n p)+g, \quad-J_{p}=\left(\nabla\left(p^{m}\right)+p \nabla \psi\right),
\end{gathered}
$$

with $x \in \Omega \subset R^{N}$, which denotes the bounded domain occupied by semiconductor crystal. Here, the unknowns $\psi, n$, and $p$ denote the electrostatic potential, the electron density, and the hole density, respectively. The $J_{n}$ represents the electron current, and $J_{p}$ is the analogously defined physical quantity of the positively charged holes. Additionally, the given function $C(x)$ denotes the doping profile (fixed charged background ions) characterizing the semiconductor under consideration, $R(n, p)=r(n, p)(1-n p)$ the net recombinationgeneration rate, and $g$ the laser density. The constants $m>0$ are the adiabatic or isothermal 
(if $m=1$ ) exponents. The regime $m>1$ describes a slow diffusion process in the electron (hole) density, whereas $0<m<1$ is related to fast diffusion.

We supplement these equations with physically motivated boundary conditions [1]:

$$
\begin{gathered}
(\psi, n, p)=\left(\psi_{D}, n_{D}, p_{D}\right), \quad(x, t) \in \Sigma_{D} \equiv \Gamma_{D} \times(0, T) \\
\left(\frac{\partial \psi}{\partial \eta}, \frac{\partial n}{\partial \eta}, \frac{\partial p}{\partial \eta}\right)=(0,0,0), \quad(x, t) \in \Sigma_{N} \equiv \Gamma_{N} \times(0, T), \\
(n, p)=\left(n_{0}, p_{0}\right), \quad x \in \Omega, t=0,
\end{gathered}
$$

where $\partial \Omega$ splits into two disjoint subsets $\Gamma_{N}$ and $\Gamma_{D} . \Gamma_{N}$ models the union of insulating boundary segments and $\Gamma_{D}$ the union of ohmic contacts.

The standard drift-diffusion model corresponding to $m=1$ has been mathematically and numerically investigated in many papers (see [1-4]). Existence and uniqueness of weak solutions have been shown. Recently, the existence analysis of the bipolar drift-diffusion problem in the adiabatic case has been studied by many authors [5-9]. But, as far as we know, few works are concerned with the mixed boundary value problem of the drift-diffusion model with the fast diffusion terms.

The phenomenon of extinction is an important property of solutions for many evolutionary equations which have been studied extensively by many authors; see, for example, $[10,11]$. Especially, there are some papers concerning the extinction for fast diffusive equations. For instance, in [12], the extinction and positivity for the evolutionary $p$-Laplacian equation without sources were studied by some authors, and $\mathrm{Li}$ and $\mathrm{Wu}$ [13] investigated the extinction for a fast diffusive filtration equation with nonlinear source terms. But, most of the work about extinction results is limited to single equation.

We prove first the global existence of weak solutions to problem (1.1)-(1.6) in the sense of the definition below. When $g \equiv 0$ and the boundary condition is homogeneous and of Dirichlet type, we show that the solutions $n(x, t), p(x, t)$ vanish in finite time in the following two cases.

(i) The initial data are appropriately small.

(ii) The doping profile is sufficiently small and generation rate grows very slowly even if the initial data are very large.

Simultaneously, the decay estimates of solutions are obtained. For the proof of our result, we employ $L^{p}$-integral model estimate method and a crucial lemma on differential inequality. This technique has been successfully applied to the porous medium equation [14].

We make the following assumptions.

(H1) $\Omega \subset R^{N}(N=1,2,3)$ is bounded and $\partial \Omega \in C^{0,1}$, whose outward normal vector is $\eta$.

(H2) $C(x), g(x) \in L^{\infty}(\Omega)$ and $g(x) \geq 0$ for a.e. $x \in \Omega$.

(H3) $r(n, p)$ is a locally Lipschitz continuous function defined for $(n, p)$ and $0 \leq r(n, p) \leq$ $\bar{r}<\infty$.

(H4) $n_{D^{\prime}}^{m} p_{D^{\prime}}^{m} \psi_{D} \in H^{1}(\Omega) \cap L^{\infty}(\Omega)$, and $n_{D}, p_{D} \geq 0$ in $\Omega$.

(H5) $n_{0}, p_{0} \in L^{\infty}(\Omega)$ and a.e. $n_{0}, p_{0} \geq 0$ in $\Omega$. 
Remark 1.1. It is obvious that (H4) implies that $n_{D}, p_{D} \in H^{1}(\Omega) \cap L^{\infty}(\Omega)$. Let

$$
Y=\left\{\omega \in H^{1}(\Omega)|\omega|_{\Gamma_{D}}=0\right\}
$$

and let $Y^{*}$ denote the dual space of $Y$.

Definition 1.2. $(\psi, n, p)$ is called the weak solution to problem (1.1)-(1.6) if $n \in n_{D}+$ $L^{2}(0, T ; Y), p \in p_{D}+L^{2}(0, T ; Y), \psi \in \psi_{D}+L^{2}(0, T ; Y), n_{t}, p_{t} \in L^{2}\left(0, T ; Y^{*}\right),\left.n\right|_{t=0}=n_{0},\left.p\right|_{t=0}=$ $p_{0}$, and there hold

$$
\begin{aligned}
& \int_{\Omega} \nabla \psi \cdot \nabla \phi \mathrm{d} x=\int_{\Omega}(p-n+C) \phi \mathrm{d} x, \quad \forall t \in(0, T), \forall \phi \in Y, \\
& \left\langle n_{t}, v\right\rangle+\int_{0}^{T} \int_{\Omega}\left(\nabla\left(n^{m}\right)-n \nabla \psi\right) \cdot \nabla v \mathrm{~d} x \mathrm{~d} t \\
& \quad=\int_{0}^{T} \int_{\Omega}[r(n, p)(1-n p)+g] v \mathrm{~d} x \mathrm{~d} t, \quad \forall v \in L^{2}(0, T ; Y), \\
& \left\langle p_{t}, v\right\rangle+\int_{0}^{T} \int_{\Omega}\left(\nabla\left(p^{m}\right)+p \nabla \psi\right) \cdot \nabla v \mathrm{~d} x \mathrm{~d} t \\
& \quad=\int_{0}^{T} \int_{\Omega}[r(n, p)(1-n p)+g] v \mathrm{~d} x \mathrm{~d} t, \quad \forall v \in L^{2}(0, T ; Y) .
\end{aligned}
$$

\section{Existence}

This section is devoted to the proof of global existence of weak solutions to problem (1.1)(1.6). We will prove the following existence theorem.

Theorem 2.1. Under hypotheses (H1)-(H4), there exists at least one weak solution of problem (1.1)(1.6).

The following lemma will be used in the proof of existence result.

Lemma 2.2 (see [9]). Let (H1) hold, $\alpha, v, F \in L^{\infty}\left(Q_{T}\right)$, and $F, v \geq 0$ for a.e. $(x, t) \in Q_{T}, \theta_{0} \in$ $L_{+}^{\infty}(\Omega), \bar{\theta} \in H^{1}(\Omega) \cap L^{\infty}(\Omega), \gamma \in\left(L^{2}\left(Q_{T}\right)\right)^{N}, \gamma \cdot \eta=0, \operatorname{div} \gamma=i \in L^{\infty}\left(Q_{T}\right)$ weakly, and $\alpha \geq \varepsilon>$ 0 . Then, there exists a unique solution $\theta$ of the following problem:

$$
\begin{gathered}
\theta_{t}-\nabla \cdot(\alpha \nabla \theta-\gamma \theta)+v \theta=F, \quad(x, t) \in Q_{T}, \\
\left.\theta\right|_{\Sigma_{D}}=\bar{\theta},\left.\quad \frac{\partial \theta}{\partial \eta}\right|_{\Sigma_{N}}=0, \quad \theta(0)=\theta_{0}, \quad x \in \Omega,
\end{gathered}
$$

such that $\theta \in L^{2}(0, T ; Y), \theta_{t} \in L^{2}\left(0, T ; Y_{0}^{*}\right)$, and

$$
0 \leq \theta \leq l e^{\lambda t} \quad \text { a.e. }(x, t) \in Q_{T}
$$

where $l=\left(\|\bar{\theta}\|_{L^{\infty}(\Omega)}+\left\|\theta_{0}\right\|_{L^{\infty}(\Omega)}+1\right)$ and $\lambda \geq\|i\|_{L^{\infty}\left(Q_{T}\right)}+\|F\|_{L^{\infty}\left(Q_{T}\right)}$. 
Our main difficulty in the proof is that problem (1.1)-(1.6) is degenerate at points where $n, p=+\infty$, and is singular at points where $n, p=0$. This difficulty leads us to consider the following auxiliary regularized problem with the initial and boundary conditions (1.4)(1.6):

$$
\begin{gathered}
-\nabla \cdot(\nabla \psi)=p_{k}-n_{k}+C(x), \\
n_{t}-\nabla \cdot\left(m\left(\frac{1}{n_{k}+\tau}\right)^{1-m} \nabla n-n \nabla \psi\right)=r\left(n_{k}, p_{k}\right)\left(1-n p_{k}\right)+g, \\
p_{t}-\nabla \cdot\left(m\left(\frac{1}{p_{k}+\tau}\right)^{1-m} \nabla p+p \nabla \psi\right)=r\left(n_{k}, p_{k}\right)\left(1-n_{k} p\right)+g
\end{gathered}
$$

where $0<\tau<1$ and $s_{k}=\min \{k, \max \{0, s\}\}$.

Now, we use the Schauder fixed point theorem [15] to prove the existence of weak solution to the above regularized problem.

Lemma 2.3. Under hypotheses (H1)-(H4), there exists at least one weak solution of problem (2.3)(2.5), under conditions (1.4)-(1.6).

Proof. Define the set $\kappa$ as

$$
\kappa=\left\{x \in L^{2}\left(Q_{T}\right) \mid x \geq 0 \text { a.e. } x \in Q_{T},\|x\|_{L^{2}\left(Q_{T}\right)} \leq R\right\} .
$$

It is obvious that $\kappa$ is a closed convex set and weakly compact in $L^{2}\left(Q_{T}\right)$. Given $\tilde{n}, \tilde{p} \in \kappa$, we consider the following problems:

$$
\begin{aligned}
& -\nabla \cdot(\nabla \psi)=\tilde{p}_{k}-\tilde{n}_{k}+C(x), \\
& \left.\psi\right|_{\Sigma_{D}}=\psi_{D},\left.\quad \frac{\partial \psi}{\partial \eta}\right|_{\Sigma_{N}}=0, \\
& n_{t}-\nabla \cdot\left(m\left(\frac{1}{\tilde{n}_{k}+\tau}\right)^{1-m} \nabla n-n \nabla \psi\right)+r\left(\tilde{n}_{k}, \tilde{p}_{k}\right) \tilde{p}_{k} n=r\left(\tilde{n}_{k}, \tilde{p}_{k}\right)+g \\
& \left.n\right|_{\Sigma_{D}}=n_{D},\left.\quad \frac{\partial n}{\partial \eta}\right|_{\Sigma_{N}}=0, \quad n(0)=n_{0}, \quad x \in \Omega \\
& p_{t}-\nabla \cdot\left(m\left(\frac{1}{\tilde{p}_{k}+\tau}\right)^{1-m} \nabla p+p \nabla \psi\right)+r\left(\tilde{n}_{k}, \tilde{p}_{k}\right) \tilde{n}_{k} p=r\left(\tilde{n}_{k}, \tilde{p}_{k}\right)+g \\
& \left.p\right|_{\Sigma_{D}}=p_{D},\left.\quad \frac{\partial p}{\partial \eta}\right|_{\Sigma_{N}}=0, \quad p(0)=p_{0}, \quad x \in \Omega
\end{aligned}
$$

We deduce the existence of a unique weak solution of (2.7) and (2.8) with the regularity $\psi \in L^{2}\left(0, T ; H^{1}(\Omega)\right) \cap L^{\infty}\left(Q_{T}\right)$ from standard theory. Set $\alpha=m\left(1 /\left(\widetilde{n}_{k}+\tau\right)\right)^{1-m}, \gamma=\nabla \psi$, 
$v=r\left(\tilde{n}_{k}, \tilde{p}_{k}\right) \tilde{p}_{k}$, and $F=r\left(\tilde{n}_{k}, \tilde{p}_{k}\right)+g$. Lemma 2.2 ensures the existence and uniqueness of weak solution of problem (2.9)-(2.10) with the regularity $n \in L^{2}\left(0, T ; H^{1}(\Omega)\right) \cap L^{\infty}\left(Q_{T}\right)$, where $L^{\infty}$ estimate of $n$ is dependent on $k, \tau$ and the known data, but independent of $R$ by (2.2). Similar results hold for problem (2.11)-(2.12).

Thus, the map

$$
S: \kappa^{2} \longrightarrow\left(L^{2}\left(Q_{T}\right)\right)^{2}, \quad(\widetilde{n}, \tilde{p}) \longmapsto(n, p)
$$

is well defined and compact. For the above given $R, S\left(\kappa^{2}\right) \hookrightarrow \kappa^{2}$ holds if we choose $R$ sufficiently large. By using the standard method (for details, see [5] or [9]), we can obtain the continuity of map $S$. Thus, existence of a fixed point of $S$ follows the weak solution of problem (2.3)-(2.5), under conditions (1.4)-(1.6).

Lemma 2.4. The weak solutions of problem (2.3)-(2.5), under conditions (1.4)-(1.6), satisfy the estimates

$$
0 \leq n(x, t), \quad p(x, t) \leq k, \quad \text { a.e. }(x, t) \in Q_{T}
$$

if one chooses $k$ such that

$$
k \geq M:=\max \left\{\sup _{\Omega}\left\{n_{0}, p_{0}\right\}, \sup _{\Sigma_{D}}\left\{n_{D}, p_{D}\right\}\right\} e^{\left(\bar{r}+\|\|\left\|_{L^{\infty}(\Omega)}+\right\| C(x) \|_{L^{\infty}(\Omega)}\right) T}
$$

Proof. Set $N=n e^{-\beta t}$ and $P=p e^{-\beta t}$; here $\beta>0$ is to be determined. Then, $(N, P)$ satisfies

$$
\begin{gathered}
N_{t}+\beta N-\nabla \cdot\left(m\left(\frac{1}{\left(e^{\beta t} N\right)_{k}+\tau}\right)^{1-m} \nabla N-N \nabla \psi\right)=e^{-\beta t}\left[r\left(n_{k}, p_{k}\right)\left(1-n p_{k}\right)+g\right], \\
P_{t}+\beta P-\nabla \cdot\left(m\left(\frac{1}{\left(e^{\beta t} P\right)_{k}+\tau}\right)^{1-m} \nabla P+P \nabla \psi\right)=e^{-\beta t}\left[r\left(n_{k}, p_{k}\right)\left(1-n_{k} p\right)+g\right], \quad(x, t) \in Q_{T}, \\
\left(e^{\beta t} N, e^{\beta t} P\right)=\left(n_{D}, p_{D}\right), \quad(x, t) \in \Sigma_{D}, \\
\left(\frac{\partial N}{\partial \eta}, \frac{\partial P}{\partial \eta}\right)=(0,0), \quad(x, t) \in \Sigma_{N}, \\
(N, P)=\left(n_{0}, p_{0}\right), \quad x \in \Omega, t=0 .
\end{gathered}
$$

To obtain the upper bound, we compare $N$ and $P$ with $z(t):=k_{0} e^{(\mu-\beta) t}$, where

$$
k_{0} \geq \max \left\{\sup _{\Omega}\left\{n_{0}, p_{0}\right\}, \sup _{\Sigma_{D}}\left\{n_{D}, p_{D}\right\}\right\}
$$


and $\mu$ such that $\mu>\beta$ is a large constant which can be determined by the following calculations. Then, we could take $(N-z)^{+}$and $(P-z)^{+}$as test functions in (2.16) and (2.17), respectively, and obtain

$$
\begin{aligned}
\frac{1}{2} \int_{\Omega}[ & \left.(N-z)^{+^{2}}(t)+(P-z)^{+^{2}}(t)\right]+\beta \int_{0}^{t} \int_{\Omega}\left[(N-z)^{+^{2}}+(P-z)^{+^{2}}\right] \\
& +\int_{0}^{t} \int_{\Omega} m\left(\frac{1}{\left(e^{\beta t} N\right)_{k}+\tau}\right)\left|\nabla(N-z)^{+}\right|^{2}+\int_{0}^{t} \int_{\Omega} m\left(\frac{1}{\left(e^{\beta t} P\right)_{k}+\tau}\right)^{1-m}\left|\nabla(P-z)^{+}\right|^{2} \\
\leq & -\mu \int_{0}^{t} \int_{\Omega} z\left[(N-z)^{+}+(P-z)^{+}\right]+\int_{0}^{t} \int_{\Omega}\left[N \cdot \nabla(N-z)^{+}-P \cdot \nabla(P-z)^{+}\right] \cdot \nabla \psi \\
& +\int_{0}^{t} \int_{\Omega} e^{-\beta t}\left[r\left(n_{k}, p_{k}\right)+g\right]\left[(N-z)^{+}+(P-z)^{+}\right] \\
\leq & -\mu \int_{0}^{t} \int_{\Omega} z\left[(N-z)^{+}+(P-N)^{+}\right]+\frac{1}{2} \int_{0}^{t} \int_{\Omega}\left(p_{k}-n_{k}+C(x)\right)\left[(N-z)^{+^{2}}-(P-z)^{+^{2}}\right] \\
& +\int_{0}^{t} \int_{\Omega}(\bar{r}+g) z\left[(N-z)^{+}(P-N)^{+}\right]+\int_{0}^{t} \int_{\Omega} z\left(p_{k}-n_{k}+C(x)\right)\left[(N-z)^{+}+(P-z)^{+}\right] \\
\leq & \left(-\mu+\bar{r}+\|g\|_{L^{\infty}(\Omega)}+\|C(x)\|_{L^{\infty}(\Omega)}\right) \int_{0}^{t} \int_{\Omega} z\left[(N-z)^{+}+(P-z)^{+}\right] \\
& +\frac{1}{2}\|C(x)\|_{L^{\infty}(\Omega)} \int_{0}^{t} \int_{\Omega}\left[(N-z)^{+^{2}}+(P-z)^{+^{2}}\right],
\end{aligned}
$$

where in the last inequality we have used

$$
\left(p_{k}-n_{k}\right)\left[(N-z)^{+}-(P-z)^{+}\right]=e^{-\beta t}\left(p_{k}-n_{k}\right)\left[\left(n-k_{0} e^{\mu t}\right)^{+}-\left(p-k_{0} e^{\mu t}\right)^{+}\right] \leq 0 .
$$

Choosing $\beta=(1 / 2)\|C(x)\|_{L^{\infty}(\Omega)}$ and $\mu \geq \bar{r}+\|g\|_{L^{\infty}(\Omega)}+\|C(x)\|_{L^{\infty}(\Omega)}$, we get

$$
\frac{1}{2} \int_{\Omega}\left[(N-z)^{+^{2}}(t)+(P-z)^{+^{2}}(t)\right]+m\left(\frac{1}{k+\tau}\right)^{1-m} \int_{0}^{t} \int_{\Omega}\left[\left|\nabla(N-z)^{+}\right|^{2}+\left|\nabla(P-z)^{+}\right|^{2}\right] \leq 0
$$

Hence, $(N-z)^{+}+(P-z)^{+}=0$ a.e. $(x, t) \in Q_{T}$; this implies $n \leq k_{0} e^{\mu t}$ and $p \leq k_{0} e^{\mu t}$. 
Thus, we obtain a weak solution of the following problem:

$$
\begin{gathered}
-\nabla \cdot(\nabla \psi)=p-n+C(x), \\
n_{t}-\nabla \cdot\left(m\left(\frac{1}{n+\tau}\right)^{1-m} \nabla n-n \nabla \psi\right)=r(n, p)(1-n p)+g \\
p_{t}-\nabla \cdot\left(m\left(\frac{1}{p+\tau}\right)^{1-m} \nabla p+p \nabla \psi\right)=r(n, p)(1-n p)+g,
\end{gathered}
$$

with the initial and boundary conditions (1.4)-(1.6).

In what follows, we give some estimates of the weak solutions to the above problem uniformly in $\tau$, which are necessary in the proof of Theorem 2.1.

Lemma 2.5. The solutions of problem (2.25)-(2.27), under conditions (1.4)-(1.6), satisfy the estimates

$$
m^{2} \int_{0}^{T} \int_{\Omega}\left[\left(\frac{1}{n+\tau}\right)^{2-2 m}|\nabla n|^{2}+\left(\frac{1}{p+\tau}\right)^{2-2 m}|\nabla p|^{2}\right] \leq C,
$$

where $C$ is independent of $\tau$.

Proof. First of all, a standard elliptic estimate gives

$$
\|\nabla \psi\|_{L^{2}\left(Q_{T}\right)} \leq C\left(1+\|n\|_{L^{2}\left(Q_{T}\right)}+\|p\|_{L^{2}\left(Q_{T}\right)}\right) \leq C .
$$

Let

$$
\varphi\left(n, n_{D}\right)=m \int_{n_{D}}^{n}\left(\frac{1}{s+\tau}\right)^{1-m} \mathrm{~d} s
$$

Since $\left.\varphi\left(n, n_{D}\right)\right|_{\Gamma_{D}}=0$ and

$$
\begin{aligned}
\int_{0}^{T} \int_{\Omega}\left|\nabla \varphi\left(n, n_{D}\right)\right|^{2} & \leq 2 m^{2} \int_{0}^{T} \int_{\Omega}\left[\left(\frac{1}{n+\tau}\right)^{2-2 m}|\nabla n|^{2}+\left(\frac{1}{n_{D}+\tau}\right)^{2-2 m}\left|\nabla n_{D}\right|^{2}\right] \\
& \leq 2 m^{2}\left(\frac{1}{\tau}\right)^{2-2 m}\left(\|n\|_{L^{2}\left(0, T ; H^{1}(\Omega)\right)}^{2}+\left\|n_{D}\right\|_{L^{2}\left(0, T ; H^{1}(\Omega)\right)}^{2}\right) \leq C(\tau),
\end{aligned}
$$

we have $\varphi\left(n, n_{D}\right) \in L^{2}(0, T ; Y)$. Then,

$$
\begin{aligned}
m^{2} \int_{0}^{T} \int_{\Omega}\left(\frac{1}{n+\tau}\right)^{2-2 m}|\nabla n|^{2}= & m^{2} \int_{0}^{T} \int_{\Omega}\left(\frac{1}{n+\tau}\right)^{1-m} \nabla n \cdot\left(\frac{1}{n_{D}+\tau}\right)^{1-m} \nabla n_{D} \\
& -\int_{0}^{T} \int_{\Omega} n_{t} \varphi\left(n, n_{D}\right)+\int_{0}^{T} \int_{\Omega} n \nabla \psi \cdot \nabla \varphi\left(n, n_{D}\right) \\
& +\int_{0}^{T} \int_{\Omega}[r(n, p)(1-n p)+g] \varphi\left(n, n_{D}\right) \\
= & I_{1}+I_{2}+I_{3}+I_{4} .
\end{aligned}
$$


By Young's inequality and (2.29), we have

$$
\begin{aligned}
I_{1} & \leq \frac{m^{2}}{4} \int_{0} \int_{\Omega}\left(\frac{1}{n+\tau}\right)^{2-2 m}|\nabla n|^{2}+\int_{0}^{T} \int_{\Omega}\left|\nabla\left(n_{D}^{m}\right)\right|^{2}, \\
I_{3} & \leq \frac{1}{8} \int_{0}^{T} \int_{\Omega}\left|\nabla \varphi\left(n, n_{D}\right)\right|^{2}+4 \int_{0}^{T} \int_{\Omega}|n \nabla \psi|^{2} \\
& \leq \frac{m^{2}}{4} \int_{0}^{T} \int_{\Omega}\left(\frac{1}{n+\tau}\right)^{2-2 m}|\nabla n|^{2}+\frac{m^{2}}{4} \int_{0}^{T} \int_{\Omega}\left|\nabla\left(n_{D}^{m}\right)\right|^{2}+4 M^{2}\|\nabla \psi\|_{L^{2}\left(Q_{T}\right)}^{2} \\
& \leq \frac{m^{2}}{4} \int_{0}^{T} \int_{\Omega}\left(\frac{1}{n+\tau}\right)^{2-2 m}|\nabla n|^{2}+C .
\end{aligned}
$$

Furthermore,

$$
\begin{aligned}
I_{4} & \leq m\left[\bar{r}\left(1+M^{2}\right)+\|g\|_{L^{\infty}(\Omega)}\right] \int_{0}^{T} \int_{\Omega}\left[\int_{0}^{n}\left(\frac{1}{s+\tau}\right)^{1-m} \mathrm{~d} s+\int_{0}^{n_{D}}\left(\frac{1}{s+\tau}\right)^{1-m} \mathrm{~d} s\right] \\
& \leq 2 m\left[\bar{r}\left(1+M^{2}\right)+\|g\|_{L^{\infty}(\Omega)}\right]\left|Q_{T}\right| \int_{0}^{M}\left(\frac{1}{s+\tau}\right)^{1-m} \mathrm{~d} s \leq C .
\end{aligned}
$$

For the term $I_{2}$, we see that

$$
\begin{aligned}
\left|I_{2}\right| & \leq\left|\int_{0}^{T} \int_{\Omega} n \varphi_{t}\left(n, n_{D}\right)\right|+\left|\int_{\Omega} n(T) \varphi\left(n, n_{D}\right)(T)\right|+\left|\int_{\Omega} n(T) \varphi\left(n, n_{D}\right)(0)\right| \\
& \leq m\left|\int_{0}^{T} \int_{\Omega} n n_{t}\left(\frac{1}{n+\tau}\right)^{1-m}\right|+4 M|\Omega| \int_{0}^{M}\left(\frac{1}{s+\tau}\right)^{1-m} \mathrm{~d} s \\
& \leq m\left|\int_{0}^{T} \int_{\Omega} \frac{\partial}{\partial t}\left(\int_{0}^{n} s\left(\frac{1}{s+\tau}\right)^{1-m} \mathrm{~d} s\right)\right|+C \\
& \leq m\left|\int_{\Omega} \int_{n_{0}(x)}^{n(x, T)} s\left(\frac{1}{s+\tau}\right)^{1-m} \mathrm{~d} s\right|+C \leq C .
\end{aligned}
$$

Inserting (2.33)-(2.36) into (2.32), we finally get

$$
m^{2} \int_{0}^{T} \int_{\Omega}\left(\frac{1}{n+\tau}\right)^{2-2 m}|\nabla n|^{2} \leq C
$$

A similar estimate holds for $p$, and then we complete the proof.

Lemma 2.6. The weak solutions $n$ and $p$ of problem (2.25)-(2.27), under conditions (1.4)-(1.6), satisfy the following estimate:

$$
\|\nabla n\|_{L^{2}\left(Q_{T}\right)},\|\nabla p\|_{L^{2}\left(Q_{T}\right)} \leq C .
$$


Proof. We will only prove that (2.38) holds for $n$. The rest can be derived similarly. Without loss of generality, we assume $0<\tau<1$. Then, we conclude that

$$
\begin{aligned}
\int_{0}^{T} \int_{\Omega}|\nabla n|^{2} & =\int_{0}^{T} \int_{\Omega}(n+\tau)^{2-2 m}\left[\left(\frac{1}{n+\tau}\right)^{1-m}|\nabla n|\right]^{2} \\
& \leq(M+1)^{2-2 m} \int_{0}^{T} \int_{\Omega}\left[\left(\frac{1}{n+\tau}\right)^{1-m}|\nabla n|\right]^{2} \leq C .
\end{aligned}
$$

Following Lemmas 2.5 and 2.6, we can easily get the following lemma.

Lemma 2.7. The weak solutions $n$ and $p$ of problem (2.25)-(2.27), under conditions (1.4)-(1.6), satisfy the following estimate:

$$
\left\|n_{t}\right\|_{L^{2}\left(0, T ; Y^{*}\right)^{\prime}}\left\|p_{t}\right\|_{L^{2}\left(0, T ; Y^{*}\right)} \leq C
$$

Proof of Theorem 2.1. Let $\left\{\psi_{\tau}, n_{\tau}, p_{\tau}\right\}$ be the sequence of solutions of problem (2.25)-(2.27), under conditions (1.4)-(1.6). By passing to a subsequence, if necessary, from Lemmas 2.42.7, we infer that

$$
\begin{gathered}
\left(\left(n_{\varepsilon}\right)_{t^{\prime}}\left(p_{\varepsilon}\right)_{t}\right) \longrightarrow\left(n_{t}, p_{t}\right) \text { weakly in } L^{2}\left(0, T ; Y^{*}\right), \\
\left(\psi_{\varepsilon}, n_{\varepsilon}, p_{\varepsilon}\right) \longrightarrow(\psi, n, p) \text { strongly in } L^{2}\left(Q_{T}\right), \quad \text { a.e. in } Q_{T}, \\
\left(\psi_{\varepsilon}, n_{\varepsilon}, p_{\varepsilon}\right) \longrightarrow(\psi, n, p) \text { weakly in } L^{2}\left(0, T ; H^{1}(\Omega)\right), \\
\left(\nabla(n+\tau)^{m}, \nabla(p+\tau)^{m}\right) \longrightarrow\left(\nabla\left(n^{m}\right), \nabla\left(p^{m}\right)\right) \text { weakly in } L^{2}\left(Q_{T}\right) .
\end{gathered}
$$

Now, we can conclude that $(\psi, n, p)$ is a weak solution of problem (1.1)-(1.6) by standard method, and then complete the proof of Theorem 2.1.

\section{Extinction}

Besides the assumptions (H1)-(H5), we need for our extinction result the following structural condition on $r(n, p)$.

(H6) $r(n, p) \leq \rho_{0}(n+p)$ for some positive constant $\rho_{0}$.

Because $r(n, p)$ denotes the generation term in the recombination-generation rate, this condition means that the generation rate grows very slowly in $(n, p)$ when $\rho_{0}$ is very small. conditions

Additionally, we replace boundary conditions (1.4)-(1.5) with homogeneous Dirichlet

$$
n=p=\psi=0, \quad x \in \partial \Omega \times(0, T) .
$$

It is obvious that the existence of weak solutions of problem (1.1)-(1.3), under conditions (3.1) and (1.6), can be obtained in easier way than the case of mixed boundary value problem. 
Before stating our main result in this section, we list the following important conditions in Theorem 3.1:

$$
\begin{aligned}
& \|C(x)\|_{L^{\infty}(\Omega)}+2 \rho_{0} \leq \frac{1}{2} C_{0}^{-2}|\Omega|^{-2 m /(m+1)+(N-2) / N} \\
& \times\left(\left\|n_{0}\right\|_{L^{m+1}(\Omega)}^{m+1}+\left\|p_{0}\right\|_{L^{m+1}(\Omega)}^{m+1}\right)^{(m-1) /(m+1)}, \\
& \left\|n_{0}\right\|_{L^{m+1}(\Omega)}^{m+1}+\left\|p_{0}\right\|_{L^{m+1}(\Omega)}^{m+1} \leq\left(\frac{C_{0}^{-2}|\Omega|^{-2 m /(m+1)+(N-2) / N}}{\|C(x)\|_{L^{\infty}(\Omega)}+2 \rho_{0}}\right)^{(1+m) /(1-m)}, \\
& \|C(x)\|_{L^{\infty}(\Omega)}+2 \rho_{0} \leq \frac{2 m s}{(m+s)^{2}} C_{0}^{-2}\left(\left\|n_{0}\right\|_{L^{s+1}(\Omega)}^{s+1}+\left\|p_{0}\right\|_{L^{s+1}(\Omega)}^{s+1}\right)^{(m-1) /(s+1)}, \\
& \left\|n_{0}\right\|_{L^{s+1}(\Omega)}^{s+1}+\left\|p_{0}\right\|_{L^{s+1}(\Omega)}^{s+1} \leq\left[\frac{4 m s C_{0}^{-2}}{(m+s)^{2}\left(\|C(x)\|_{L^{\infty}(\Omega)}+2 \rho_{0}\right)}\right]^{(1+s) /(1-m)},
\end{aligned}
$$

where $s=(N(1-m) / 2)-1$ and $C_{0}$ is the embedding constant of the following inequality:

$$
\|u\|_{L^{2 N /(N-2)(\Omega)}} \leq C_{0}\|\nabla u\|_{L^{2}(\Omega)}, \quad \forall u \in H_{0}^{1}(\Omega) .
$$

Theorem 3.1. Let (H1)-(H6) be fulfilled, let $g(x) \equiv 0$, and let $(\psi, n, p)$ be a weak solution of problem (1.1)-(1.3), under conditions (3.1) and (1.6). Then, one has the following.

(i) If (3.2) or (3.3) below is satisfied, then $u(x, t)$ and $p(x, t)$ vanish in the finite time for $(N-2) /(N+2) \leq m<1$, and

$$
\begin{aligned}
& \|n(\cdot, t)\|_{L^{m+1}(\Omega)}^{m+1}+\|p(\cdot, t)\|_{L^{m+1}(\Omega)}^{m+1} \leq B_{1} e^{-\alpha_{1} t}, \quad t \in\left[0, T_{01}\right), \\
& \|n(\cdot, t)\|_{L^{m+1}(\Omega)}^{m+1}+\|p(\cdot, t)\|_{L^{m+1}(\Omega)}^{m+1} \\
& \quad \leq\left[\left(\left\|n\left(\cdot, T_{01}\right)\right\|_{L^{m+1}(\Omega)}^{m+1}+\left\|p\left(\cdot, T_{01}\right)\right\|_{L^{m+1}(\Omega)}^{m+1}\right)^{(1-m) /(1+m)}-\frac{C_{1}(1-m)}{1+m} t\right]^{(1+m) /(1-m)}, \\
& \quad t \in\left[T_{01}, T_{1}\right),
\end{aligned}
$$

$\|n(\cdot, t)\|_{L^{m+1}(\Omega)}^{m+1}+\|p(\cdot, t)\|_{L^{m+1}(\Omega)}^{m+1} \equiv 0, \quad t \in\left[T_{1}, \infty\right)$.

(ii) If (3.4) or (3.5) below is satisfied, then $u(x, t)$ and $p(x, t)$ vanish in the finite time for $0<m<(N-2) /(N+2)$, and

$\|n(\cdot, t)\|_{L^{s+1}(\Omega)}^{s+1}+\|p(\cdot, t)\|_{L^{s+1}(\Omega)}^{s+1} \leq B_{2} e^{-\alpha_{2} t}, \quad t \in\left[0, T_{02}\right)$,

$\|n(\cdot, t)\|_{L^{s+1}(\Omega)}^{s+1}+\|p(\cdot, t)\|_{L^{s+1}(\Omega)}^{s+1}\|n(\cdot, t)\|_{L^{s+1}(\Omega)}^{s+1}+\|p(\cdot, t)\|_{L^{s+1}(\Omega)}^{s+1}$

$\leq\left[\left(\left\|n\left(\cdot, T_{02}\right)\right\|_{L^{s+1}(\Omega)}^{s+1}+\left\|p\left(\cdot, T_{02}\right)\right\|_{L^{s+1}(\Omega)}^{s+1}\right)^{(1-m) /(1+s)}-\frac{C_{2}(1-m)}{1+s} t\right]^{(1+s) /(1-m)}$,

$t \in\left[T_{02}, T_{2}\right)$,

$\|n(\cdot, t)\|_{L^{s+1}(\Omega)}^{s+1}+\|p(\cdot, t)\|_{L^{s+1}(\Omega)}^{s+1} \equiv 0, \quad t \in\left[T_{2}, \infty\right)$.

Here, $C_{1}, C_{2}, T_{1}$, and $T_{2}$ will be given by (3.22), (3.32), (3.25), and (3.33), respectively. 
To obtain the above result, we will use the following lemma which is of crucial importance to the proof.

Lemma 3.2. Let $0<k<q \leq 1$, and let $y(t) \geq 0$ be a solution of the differential inequality

$$
\frac{\mathrm{d} y}{\mathrm{~d} t}+\beta y^{k} \leq \sigma y^{q} \quad(t \geq 0), y(0)=y_{0} \geq 0,
$$

where $\beta$ and $\sigma$ are positive constants. If

$$
\text { (i) } y_{0}<\left(\frac{\beta}{\sigma}\right)^{1 /(q-k)} \text { or } \text { (ii) } \sigma \leq \frac{\beta y_{0}^{k-q}}{2}
$$

holds, then there exist $\alpha>0$ and $B>0$ such that

$$
0 \leq y(t) \leq B e^{-\alpha t}, \quad t \geq 0 .
$$

Proof. We will use upper and lower solutions' methods to complete the proof. The proof of case (i) is basically the same as method of case (ii). So, we are only devoted to the proof of case (i). In fact, we only need to choose $\alpha, B$ properly such that $B e^{-\alpha t}$ is an upper solution of (3.9). That is, $\alpha$ and $B$ must satisfy

$$
-\alpha B e^{-\alpha t}+\beta B^{k} e^{-\alpha k t} \geq \sigma B^{q} e^{-\alpha q t} \quad(t \geq 0), B \geq y_{0} .
$$

Choose $B$ such that $\ln B<\ln (\beta / \sigma) /(q-k)$ and $\alpha$ sufficiently small. Then,

$$
\frac{\sigma}{\beta} B^{q-k}+\frac{\alpha}{\beta} B^{1-k} \leq 1
$$

which implies that

$$
\alpha(q-k) t \geq \ln \left(\frac{\sigma}{\beta} B^{q-k}+\frac{\alpha}{\beta} B^{1-k}\right), \quad \forall t \geq 0 .
$$

Noting that $0<q \leq 1$, therefore we have

$$
e^{-\alpha(k-q) t} \geq \frac{\sigma}{\beta} B^{q-k}+\frac{\alpha}{\beta} B^{1-k} e^{-(1-q) \alpha t}, \quad \forall t \geq 0,
$$

which is equivalent to (3.12) if $y_{0} \leq B<(\beta / \sigma)^{1 /(q-k)}$. The lemma is proved.

Proof of Theorem 3.1. We consider first the case $(N-2) /(N+2) \leq m<1$. Multiplying (1.2) and (1.3) by $n^{m}$ and $p^{m}$, respectively, and employing the Poisson equation (1.1), we get

$$
\begin{aligned}
\frac{1}{m+1} & \frac{\mathrm{d}}{\mathrm{d} t} \int_{\Omega}\left(n^{m+1}+p^{m+1}\right) \mathrm{d} x+\int_{\Omega}\left[\left|\nabla\left(n^{m}\right)\right|^{2}+\left|\nabla\left(p^{m}\right)\right|^{2}\right] \mathrm{d} x \\
\quad & \frac{m}{m+1} \int_{\Omega}(p-n+C(x))\left(n^{m+1}+p^{m+1}\right) \mathrm{d} x+\int_{\Omega} r(n, p)(1-n p)\left(n^{m}+p^{m}\right) \mathrm{d} x \\
\quad & I_{1}+I_{2} .
\end{aligned}
$$


The embedding theorem gives

$$
\begin{aligned}
\|n\|_{L^{m+1}(\Omega)}^{m} & \leq|\Omega|^{m /(m+1)-(N-2) / 2 N}\left\|n^{m}\right\|_{L^{2 N /(N-2)(\Omega)}} \\
& \leq C_{0}|\Omega|^{m /(m+1)-(N-2) / 2 N}\left\|\nabla\left(n^{m}\right)\right\|_{L^{2}(\Omega)}
\end{aligned}
$$

and similarly for $p$.

By Young's inequality, we have

$$
I_{2} \leq \rho_{0} \int_{\Omega}(n+p)\left(n^{m}+p^{m}\right) \mathrm{d} x \leq 2 \rho_{0} \int_{\Omega}\left(n^{m+1}+p^{m+1}\right) \mathrm{d} x .
$$

By (3.16)-(3.18), taking into account $(p-n)\left(n^{m+1}-p^{m+1}\right) \leq 0$, we obtain

$$
\frac{\mathrm{d}}{\mathrm{d} t} f_{1}(t)+(m+1) C_{0}^{-2}|\Omega|^{-2 m /(m+1)+(N-2) / N} f_{1}(t)^{2 m /(m+1)} \leq(m+1)\left(\|C(x)\|_{L^{\infty}(\Omega)}+2 \rho_{0}\right) f_{1}(t),
$$

where

$$
f_{1}(t)=\|n\|_{L^{m+1}(\Omega)}^{m+1}+\|p\|_{L^{m+1}(\Omega)}^{m+1} .
$$

By Lemma 3.2, there exist $\alpha_{1}$ and $B_{1}$ such that

$$
0 \leq f_{1}(t) \leq B_{1} e^{-\alpha_{1} t}, \quad t \geq 0,
$$

if (3.2) or (3.3) holds.

Furthermore, there exist $T_{01}$ such that

$$
\begin{aligned}
(1+m) & {\left[C_{0}^{-2}|\Omega|^{-2 m /(m+1)+(N-2) / N}-\left(\|C(x)\|_{L^{\infty}(\Omega)}+2 \rho_{0}\right) f_{1}(t)^{(1-m) /(1+m)}\right] } \\
\geq & (1+m)\left[C_{0}^{-2}|\Omega|^{-2 m /(m+1)+(N-2) / N}-\left(\|C(x)\|_{L^{\infty}(\Omega)}+2 \rho_{0}\right)\left(B_{1} e^{-\alpha_{1} T_{01}}\right)^{(1-m) /(1+m)}\right] \\
& :=C_{1}>0
\end{aligned}
$$

holds for $t \in\left[T_{01}, \infty\right)$. Thus, when $t \in\left[T_{01}, \infty\right)$, (3.19) turns to

$$
\frac{\mathrm{d}}{\mathrm{d} t} f_{1}(t)+C_{1} f_{1}(t)^{2 m /(m+1)} \leq 0, \quad t \geq T_{01} .
$$

By a direct calculation, we deduce that

$$
\begin{aligned}
& f_{1}(t) \leq\left[f_{1}\left(T_{01}\right)^{(1-m) /(1+m)}-\frac{C_{1}(1-m)}{1+m} t\right]^{(1+m) /(1-m)}, t \in\left[T_{01}, T_{1}\right), \\
& f_{1}(t) \equiv 0, \quad t \in\left[T_{1}, \infty\right)
\end{aligned}
$$

where

$$
T_{1}=\frac{1+m}{C_{1}(1-m)}\left(\left\|n\left(\cdot, T_{01}\right)\right\|_{L^{m+1}(\Omega)}^{m+1}+\left\|p\left(\cdot, T_{01}\right)\right\|_{L^{m+1}(\Omega)}^{m+1}\right)^{(1-m) /(1+m)}
$$


For the case $0<m<(N-2) /(N+2)$, using $n^{s}$ and $p^{s}$ as test functions in (1.2) and (1.3), respectively, we obtain

$$
\begin{aligned}
\frac{1}{s+1} & \frac{\mathrm{d}}{\mathrm{d} t} \int_{\Omega}\left(n^{s+1}+p^{s+1}\right) \mathrm{d} x+\frac{4 m s}{(m+s)^{2}} \int_{\Omega}\left[\left|\nabla\left(n^{(m+s) / 2}\right)\right|^{2}+\left|\nabla\left(p^{(m+s) / 2}\right)\right|^{2}\right] \mathrm{d} x \\
\quad & \frac{s}{s+1} \int_{\Omega}(p-n+C(x))\left(n^{s+1}+p^{s+1}\right) \mathrm{d} x+\int_{\Omega} r(n, p)(1-n p)\left(n^{s}+p^{s}\right) \mathrm{d} x .
\end{aligned}
$$

To simplify, we denote

$$
f_{2}(t)=\|n\|_{L^{s+1}(\Omega)}^{s+1}+\|p\|_{L^{s+1}(\Omega)}^{s+1} .
$$

Hence, we have

$$
\frac{\mathrm{d}}{\mathrm{d} t} f_{2}(t)+\frac{4(s+1) m s}{(m+s)^{2}} C_{0}^{-2} f_{2}(t)^{(m+s) /(1+s)} \leq(s+1)\left(\|C(x)\|_{L^{\infty}(\Omega)}+2 \rho_{0}\right) f_{2}(t),
$$

where we have used

$$
\|u\|_{L^{s+1}(\Omega)}^{(m+s) / 2}=\left(\int_{\Omega} u^{(m+s) / 2 \cdot 2 N /(N-2)} \mathrm{d} x\right)^{(N-2) / 2 N} \leq C_{0}\left\|\nabla\left(u^{(m+s) / 2}\right)\right\|_{L^{2}(\Omega)^{\prime}} \quad \forall u \in H_{0}^{1}(\Omega) .
$$

By Lemma 3.2, there exist $\alpha_{2}$ and $B_{2}$ such that

$$
0 \leq f_{2}(t) \leq B_{2} e^{-\alpha_{2} t}, \quad t \geq 0,
$$

if (3.4) or (3.5) holds.

By a simple analysis similar to that of the case $(N-2) /(N+2) \leq m<1$, we obtain

$$
\begin{aligned}
& f_{2}(t) \leq\left[f_{2}\left(T_{02}\right)^{(1-m) /(1+s)}-\frac{C_{2}(1-m)}{1+s} t\right]^{(1+s) /(1-m)}, \quad t \in\left[T_{02}, T_{2}\right), \\
& f_{2}(t) \equiv 0, \quad t \in\left[T_{2}, \infty\right),
\end{aligned}
$$

where

$$
\begin{aligned}
& C_{2}=(s+1)\left[\frac{4 m s}{(m+s)^{2}} C_{0}^{-2}-\left(\|C(x)\|_{L^{\infty}(\Omega)}+2 \rho_{0}\right)\left(B_{2} e^{-\alpha_{2} T_{02}}\right)^{(1-m) /(1+s)}\right]>0, \\
& T_{2}=\frac{1+s}{C_{2}(1-m)}\left(\left\|n\left(\cdot, T_{02}\right)\right\|_{L^{s+1}(\Omega)}^{s+1}+\left\|p\left(\cdot, T_{02}\right)\right\|_{L^{s+1}(\Omega)}^{s+1}\right)^{(1-m) /(1+s)} .
\end{aligned}
$$

\section{References}

[1] P. A. Markowich, C. A. Ringhofer, and C. Schmeiser, Semiconductor Equations, Springer, Vienna, Austria, 1990.

[2] H. Gajewski, "On existence, uniqueness and asymptotic behavior of solutions of the basic equations for carrier transport in semiconductors," Zeitschrift für Angewwandte Mathematik und Mechanik, vol. 65, no. 2, pp. 101-108, 1985. 
[3] W. F. Fang and K. Ito, "On the time-dependent drift-diffusion model for semiconductors," Journal of Differential Equations, vol. 117, no. 2, pp. 245-280, 1995.

[4] W. F. Fang and K. Ito, "Global solutions of the time-dependent drift-diffusion semiconductor equations," Journal of Differential Equations, vol. 123, no. 2, pp. 523-566, 1995.

[5] A. Jüngel, "On the existence and uniqueness of transient solutions of a degenerate nonlinear driftdiffusion model for semiconductors," Mathematical Models $\mathcal{E}$ Methods in Applied Sciences, vol. 4, no. 5, pp. 677-703, 1994.

[6] A. Jüngel, "Qualitative behavior of solutions of a degenerate nonlinear drift-diffusion model for semiconductors," Mathematical Models \& Methods in Applied Sciences, vol. 5, no. 4, pp. 497-518, 1995.

[7] J. I. Díaz, G. Galiano, and A. Jüngel, "On a quasilinear degenerate system arising in semiconductors theory-part I: existence and uniqueness of solutions," Nonlinear Analysis: Real World Applications, vol. 2, no. 3, pp. 305-336, 2001.

[8] J. I. Díaz, G. Galiano, and A. Jüngel, “On a quasilinear degenerate system arising in semiconductor theory-part II: localization of vacuum solutions," Nonlinear Analysis: Theory, Methods E Applications, vol. 36, no. 5, pp. 569-594, 1999.

[9] P. Guan and B. Wu, "Existence of weak solutions to a degenerate time-dependent semiconductor equations with temperature effect," Journal of Mathematical Analysis and Applications, vol. 332, no. 1, pp. 367-380, 2007.

[10] S. N. Antontsev and S. I. Shmarev, "A model porous medium equation with variable exponent of nonlinearity: existence, uniqueness and localization properties of solutions," Nonlinear Analysis: Theory, Methods E Applications, vol. 60, no. 3, pp. 515-545, 2005.

[11] R. Ferreira and J. L. Vazquez, "Extinction behaviour for fast diffusion equations with absorption," Nonlinear Analysis: Theory, Methods \& Applications, vol. 43, no. 8, pp. 943-985, 2001.

[12] H. Yuan, S. Lian, W. Gao, X. Xu, and C. Cao, “Extinction and positivity for the evolution $p$-Laplacian equation in $\mathrm{R}^{n}, "$ Nonlinear Analysis: Theory, Methods \& Applications, vol. 60, no. 6, pp. 1085-1091, 2005.

[13] Y. Li and J. Wu, "Extinction for fast diffusion equations with nonlinear sources," Electronic Journal of Differential Equations, vol. 2005, no. 23, pp. 1-7, 2005.

[14] W. Liu, M. Wang, and B. Wu, "Extinction and decay estimates of solutions for a class of porous medium equations," Journal of Inequalities and Applications, vol. 2007, Article ID 87650, 8 pages, 2007.

[15] O. A. Ladyžhenskaya and N. N. Vral'tseva, Linear and Quasilinear Elliptic Equations, Academic Press, New York, NY, USA, 1968. 